\title{
Longitudinal evaluation of markers of hemostasis in pregnancy
}

\author{
Hammerova $\mathrm{L}^{1}$, Chabada $\mathrm{J}^{1}$, Drobny $\mathbf{J}^{1}$, Batorova $\mathrm{A}^{2}$ \\ 1st Department of Gynaecology and Obstetrics of Comenius University Bratislava, Slovakia. \\ hammerova@gmail.com
}

\begin{abstract}
Objective: The aim of this study was to establish the physiologic changes in hemostasis during pregnancy and to find the association between the factor $\mathrm{V}$ Leiden mutation and adverse pregnancy outcome. Methods: We investigated blood samples of 148 pregnant women during each trimester of pregnancy. We measured their serum concentrations of factors I, II, V, VII, VIII, IX, X, XI, XII, D-dimers, prothrombin time, INR, aPTT, activity of protein $\mathrm{C}$ and $\mathrm{S}$, antithrombin III and platelet count. The pregnancy outcome of women with factor $\mathrm{V}$ Leiden mutation was compared to those without congenital thrombophilia.

Results: Prothrombin time, INR and aPTT were significantly shorter. We found significantly higher plasma concentrations of fibrinogen and d-dimers and higher levels of activity of factor VII and X in the third trimester. No significant difference was found in protein $\mathrm{C}$ and antithrombin III activity. The protein S activity was lower in the second trimester and it increased in the third trimester. Although most of the clotting factors were rising during the pregnancy, there was no evidence of fibrinolytic overactivation. In our study, the carriership of factor $\mathrm{V}$ Leiden mutation did not affect the incidence of preeclampsia, eclampsia, intrauterine fetal death and venous thromboembolism. Placental abruption was rare.

Conclusion: Hemostatic changes in pregnancy are significant and essential, and have the potential to cause adverse pregnancy outcome. In addition, hypercoagulable state during pregnancy is considered to be physiological (Tab. 4, Ref. 36). Text in PDF www.elis.sk.

Key words: pregnancy, hemostasis, hypercoagulable state, venous thromboembolism.
\end{abstract}

Pregnancy is considered to be a hypercoagulable state with higher risk of thromboembolic events. During pregnancy, changes in the hemostasis are involved in increasing the stasis and coagulation factors and decreasing the levels of anticoagulant proteins. The physiological activation of hemostasis during pregnancy reflects the venous stasis, vascular damage and hypercoagulation (1).

Pulmonary embolism is the main cause of maternal mortality in the developed countries (2). The incidence of deep venous thrombosis varies from 1:1000 to 1:2000 deliveries and rises during the third trimester and in the puerperium (2). The risk factors for venous thromboembolism differ from those for arterial disease, particularly stroke and atherogenic factors such as smoking, hypertension or hyperlipidemia. The major generally known risk factors for venous thrombosis in pregnancy are venous stasis and hypercoagulation (3). Another classification dividing the risk factors into acquired and inherited conditions is shown in Table 1 (3).

1st Department of Gynaecology and Obstetrics of Comenius University, Bratislava, Slovakia

Address for correspondence: L. Hammerova, MD, 1st Department of Gynaecology and Obstetrics of Comenius University, Antolska 11, SK85107 Bratislava, Slovakia.

Phone: +421.918522079

Acknowledgement: Authors thank for responsible work of The National Hemophilia Centre at The Institute of Hematology and Blood Transfusion, University Hospital, Bratislava, Slovakia.
Tab. 1. Risk factors of deep venous thrombosis in pregnancy (3).

\begin{tabular}{l|l|l}
\hline Acquired & Inherited & Mixed \\
\hline Age & Factor V Leiden & Hyperhomocysteinemia \\
Previous thrombosis & Protein S deficiency & Hyperfibrinogenemia \\
Immobilization & Protein C deficiency & High levels of factor \\
Surgery & Antithrombin defi- & VIII \\
Trauma & ciency & \\
Malignancy & Factor II gene muta- & \\
Antiphospholipid syn- & tion G20210A & \\
drome & Dysfibrinogenemia & \\
Myeloproliferative & & \\
disorders & & \\
Venous stasis & & \\
Obesity & \\
Vascular abnormalities & \\
Nephrotic syndrome & \\
Sepsis & & \\
\hline
\end{tabular}

During pregnancy, the concentration of most clotting factors increases, some of the natural anticoagulants decrease, and fibrinolytic activity diminishes. The gentle balance shifts towards enhanced coagulation. The modifications of the coagulation system are due to hormonal changes while on the one hand they protect women from excessive bleeding and fatal hemorrhage during delivery and puerperium, but on the other they predispose them to thromboembolism (4). The most interesting markers of hemosta- 
sis activation and thrombin generation are antithrombin III and D-dimer. Together with fibrinogen levels and platelet counts, the activation markers are useful tools in various pathological situations in pregnancy for predicting and monitoring the severity of the condition (5).

Both congenital and acquired thrombophilias are implicated in pathophysiological processes associated with thrombotic damage of the placenta and associated with increased risk of venous thromboembolism. Activated protein $\mathrm{C}$ resistance caused by factor $\mathrm{V}$ Leiden mutation is the most commonly known inherited risk factor for venous thrombosis in pregnancy in white European population. The gene for factor V Leiden mutation results in replacement of arginine at position 506 by glutamine. This causes impaired anticoagulation. The resulting hypercoagulable state is a lifelong risk factor for venous thrombosis and rises during pregnancy. The combination of activated protein $\mathrm{C}$ resistence/factor $\mathrm{V}$ Leiden mutation and pregnancy may predict a higher risk for thromboembolic phenomena and adverse pregnancy outcome (2). Thrombotic processes have been described as an important pathogenetic factor of some severe obstetric conditions such as preeclampsia, intrauterine growth restriction (IUGR) and placental abruption by influencing the placental perfusion. Preeclampsia, placental abruption, intrauterine growth restriction (IUGR) and intrauterine fetal death (IUFD) contribute to maternal and fetal morbidity and mortality. Preeclampsia might be caused by poor placentation involving the spiral arteries and leads to inadequate uteroplacental flow. Between the 8th and 18th gestation weeks, an altered trophoblast invasion of the spiral arterial walls appears and results in massive maternal inflammatory response. Placental changes characterized by superficial endovascular cytotrophoblast invasion in the spiral arteries and intervillous space are referred to as placental vasculopathy. Placental apoptotic and necrotic debris reaches maternal circulation resulting in endothelial damage and activation of blood coagulation (6). Placental abruption is a serious obstetric condition associated with high perinatal mortality and high maternal morbidity and mortality. Abruption of placenta is characterized as a separation of implanted placenta by hemorrhage into decidua basalis. This process starts with uterine vasospasm followed by relaxation and consequent venous stasis. The arterioles rupture and placenta separates. Placental biopsies after abruption show the same abnormal vascular structures as in preeclampsia (7). Intrauterine growth restriction (IUGR) has serious consequences for neonates. IUGR is associated with increased risk of premature birth, increased morbidity including necrotizing enterocolitis, hypoxic brain injury, retinopathy of prematurity, in later life obesity, diabetes and ischemic heart diseases. Placenta is the interface between fetal and maternal circulation and plays an essential role in supporting normal fetal growth. Placental pathology is responsible for the majority of IUGR (8). The relationship between intrauterine fetal death (IUFD) and factor V mutation is still debatable (9). It is unlikely that the factor V Leiden mutation can cause early miscarriage by increasing the blood coagulation, because until the 10th week of pregnancy, the fetal nutrition is mainly histiotrophic and the maternal intraplacental circulation is fully established after the third month of gestation (9).

The aim of this study was to evaluate changes in plasma levels of many hemostatic variables between 10th and 36th weeks during pregnancy and to find the association between the factor V Leiden mutation and adverse pregnancy outcomes such as preeclampsia, eclampsia, intrauterine growth restriction (IUGR), intrauterine fetal death (IUFD), prematurity, placental abruption and venous thromboembolism.

\section{Methods}

In our prospective study, we investigated 148 pregnant women during pregnancy between October 2006 and September 2009. All women in the study population were Caucasian. This study included only women with singleton pregnancy and history of one unexplained abortion in the first trimester. Women with obesity (BMI $>40)$, liver and kidney diseases and other diseases that could possibly affect the coagulation parameters were excluded from the investigation. The women were non-smokers. All subjects gave informed consent according to the principals of the Declaration of Helsinki.

The blood samples were obtained at 10th-15th, 20th-24th and 32 nd -36 th weeks of pregnancy and the hemostasis markers were compared in each trimester. Venous blood samples were obtained in fasting state using dehydrated sodium citrate for blood clotting studies, and the samples were centrifuged at $3500 \mathrm{x}$ g for 15 minutes at room temperature $\left(24^{\circ} \mathrm{C}\right)$. The obtained plasma was measured by fully automatic coagulometer BCS XP Dade Behring. Plasma was collected and assays were performed as follows:

- Global tests: prothrombin time (PT) also expressed in INR (International Normalized Ratio), activated partial thromboplastin time (aPTT) and platelets count.

- Coagulation factors: factor I, factor II, factor V, factor VII, factor VIII, factor IX, factor X, factor XI and factor XII.

- Fibrinolysis marker: D-dimer in plasma measured by ELISA

- Natural inhibitors of coagulation: protein $\mathrm{C}$, protein $\mathrm{S}$ and antithrombin III.

In our study group, the diagnosis of factor V Leiden mutation was made in the pre-conception period in 52 women. The heterozygous factor V Leiden mutation was confirmed in 11 women by polymerase chain reaction (PCR). Negative results were obtained in 41 women. Five women with factor V Leiden mutation were treated during pregnancy with a prophylactic dose of Dalteparin. We observed these women from the 10th-15th week of pregnancy until their delivery. The factor V Leiden carriers were compared with controls with negative PCR in respect of gestational age at delivery, preeclampsia, eclampsia, intrauterine fetal death, intrauterine growth restriction, placental abruption, blood loss at delivery, frequency of venous thromboembolism and superficial thrombophlebitis. A paired t-test for normally distributed data was used for comparing the investigated groups. A significance level of 0.05 or less was considered statistically significant. 
$140-144$

\section{Results}

The changes in coagulation and fibrinolytic factors during pregnancy are shown in Table 2. When examining the global test, we found prothrombin time, INR and activated partial thromboplastin time to be significantly shorter. When we compared procoagulant factors in the second and third trimesters with those in the first trimester, we found significantly higher plasma concentrations of fibrinogen and D-dimers and higher levels of activity of factors VII and X. No significant difference was found in protein $\mathrm{C}$ and antithrombin III activity. The protein S activity was lower in the second trimester and increased in the third trimester.

Data of pregnancy outcome in women with factor $\mathrm{V}$ Leiden mutation and controls are shown in Table 3. All pregnancies ended with delivery of a living infant. None of the 52 pregnancies were complicated by venous thromboembolism. More factor V Leiden mutation carriers than controls were given thromboprophylaxis and compression stockings, which might have prevented some VTE (five women with factor V Leiden mutation were treated with Dalteparin and one had compression stockings because of varicose veins of lower extremities). There were significant differences re-

Tab. 2. Hemostatic changes during pregnancy.

\begin{tabular}{|c|c|c|c|c|}
\hline \multirow{2}{*}{$\begin{array}{l}\text { Variables } \\
(\text { mean } \pm \mathrm{SD})\end{array}$} & \multicolumn{3}{|c|}{ Week of Pregnancy } & \multirow{2}{*}{$\begin{array}{c}\text { Normal } \\
\text { range }\end{array}$} \\
\hline & $10-15$ & $20-24$ & $32-36$ & \\
\hline Prothrombintime(\%) & $103.6 \pm 18.82$ & $102.9 \pm 18.05$ & $101.6 \pm 17.79$ & $70-130$ \\
\hline INR & $1.002 \pm 0.113$ & $0.893 \pm 0.138$ & $0.821 \pm 0.146$ & $0.8-1.25$ \\
\hline aPTT (seconds) & $31.27 \pm 3.273$ & $30.87 \pm 3.133$ & $30.43 \pm 3.201$ & $26-36$ \\
\hline Platelets $\left(\times 10^{9} \cdot 1^{-1}\right)$ & $253.4 \pm 76.05$ & $239.0 \pm 72.89$ & $228.0 \pm 74.54$ & $150-440$ \\
\hline Fibrinogen (g.1-1) & $2.969 \pm 0.774$ & $3.890 \pm 1.143$ & $4.967 \pm 1.272$ & $1.8-3.6$ \\
\hline Factor II $(\%)$ & $93.80 \pm 15.25$ & $93.83 \pm 15.21$ & $93.73 \pm 15.21$ & $70-120$ \\
\hline Factor V $(\%)$ & $103.6 \pm 21.03$ & $103.5 \pm 21.07$ & $103.7 \pm 21.04$ & $70-140$ \\
\hline Factor VII (\%) & $98.17 \pm 11.22$ & $122.6 \pm 14.92$ & $151.6 \pm 17.67$ & $70-120$ \\
\hline Factor VIII (\%) & $105.8 \pm 22.73$ & $106.2 \pm 22.74$ & $106.7 \pm 22.75$ & $70-150$ \\
\hline Factor IX (\%) & $97.43 \pm 15.03$ & $97.18 \pm 15.05$ & $97.92 \pm 15.02$ & $70-120$ \\
\hline Factor X $(\%)$ & $95.70 \pm 14.23$ & $97.99 \pm 14.18$ & $126.6 \pm 15.96$ & $70-120$ \\
\hline Factor XI (\%) & $93.16 \pm 13.99$ & $92.95 \pm 13.98$ & $92.72 \pm 13.93$ & $70-120$ \\
\hline Factor XII (\%) & $109.1 \pm 21.85$ & $109.7 \pm 21.94$ & $110.1 \pm 21.96$ & $70-150$ \\
\hline Protein C (\%) & $104.0 \pm 19.33$ & $104.0 \pm 19.30$ & $104.0 \pm 19.35$ & $70-140$ \\
\hline Protein S (\%) & $89.58 \pm 18.18$ & $85.48 \pm 18.52$ & $87.70 \pm 18.32$ & $59-118$ \\
\hline Antithrombin (\%) & $101.3 \pm 13.57$ & $101.3 \pm 13.61$ & $101.4 \pm 13.67$ & $75-125$ \\
\hline D-dimers $\left(\mu \mathrm{g} .1^{-1}\right)$ & $165.1 \pm 93.93$ & $496.2 \pm 184.3$ & $979.0 \pm 611.2$ & $0-470$ \\
\hline
\end{tabular}

Tab. 3. Pregnancy outcome in women with heterozygous factor $\mathbf{V}$ Leiden (carriers) and controls.

\begin{tabular}{lcc}
\hline & Carriers & Controls \\
\hline Amount of patients & 11 & 41 \\
Viable infants $(\% *)$ & $11(100)$ & $41(100)$ \\
IUFD $(\% *)$ & 0 & 0 \\
IUGR $(\% *)$ & $3(27.3)$ & $4(9.7)$ \\
Preeclampsia $(\% *)$ & $1(9.1)$ & $6(14.6)$ \\
Eclampsia $(\% *)$ & 0 & 0 \\
Birth before 37 th week $(\% *)$ & $1(9.1)$ & $7(17.1)$ \\
Placental abruption $(\% *)$ & $1(9.1)$ & 0 \\
Venous thromboembolism $(\% *)$ & 0 & 0 \\
Vaginal delivery $(\% *)$ & $3(27.3)$ & $25(60.9)$ \\
Caesarean section $(\% *)$ & $8(72.7)$ & $16(39.1)$ \\
$*$ - percent of pregnancies, IUFD - intrauterine fetal death, IUGR - intrauterine \\
growth retardation
\end{tabular}

Tab. 4. Comparison of total blood loss in carriers and controls during delivery.

\begin{tabular}{lcc}
\hline Blood loss $(\mathrm{ml})$ & Amount of carriers $(\% *)$ & Amount of controls $(\% *)$ \\
\hline$>1000$ & 0 & $2(4.8)$ \\
$600-1000$ & $3(27.3)$ & $11(26.8)$ \\
$<600$ & $8(72.7)$ & $28(68.4)$ \\
\hline
\end{tabular}

*-percent of pregnancies

garding placenta-mediated complications. The gestational age at delivery showed significant differences $(\mathrm{p}<0.05)$. There was a significant difference in birth weight deviation between FVL carriers and controls $(\mathrm{p}<0.05)$. The total blood loss at delivery defined by measuring and guessing was lower in the factor V Leiden carriers, but the difference was not significant. The incidence of blood loss exceeding $1000 \mathrm{ml}$ was higher among the control group $(\mathrm{p}<0.05)$. Table 4 shows the comparison of total blood loss during delivery between factor $\mathrm{V}$ Leiden mutation carriers and controls.

\section{Discussion}

The changes in hemostatic variables reflect the coagulation status and show that the pregnancy is associated with a continual low-grade process of intravascular coagulation. Our study found $8.8 \%$ of women with platelet count lower then $150 \times 10^{9} 1^{-1}$ in the first trimester. The incidence of thrombocytopenia increased in the second $(11.48 \%)$ and third (14.19\%) trimesters. We confirmed that thrombocytopenia is the most commonly identified coagulopathy in pregnancy. Some studies pointed out the benign nature of incidental thrombocytopenia in pregnancy $(10,11)$. The physiological pregnancy is associated with a degree of enhanced platelet destruction followed by compensative increased production. Sainio reported that $7.3 \%$ of full-term women involved in one-year-lasting study had platelet counts lower than $150 \times 10^{9} 1^{-1}$ at delivery (11). The prothrombin time and activated partial thromboplastin time were tested separately to test the two pathways of the clotting system, i.e. the intrinsic and extrinsic pathways. The shortening of prothrombin time was progressive and statistically significant in each trimester. This shortening was probably the consequence of increased levels of factor VII. Significant changes were captured in levels of factor IX, XI and XII during pregnancy and this might be the reason why activated partial thromboplastin time was shorter. The marked increase in plasma fibrinogen was statistically significant from the first trimester probably due to increased hepatic synthesis, and rose steadily throughout pregnancy. This could explain the almost two-fold increase in the third trimester when compared to the first trimester. The rise in fibrinogen levels reflects the increased production $(12,13)$. The significant indicators of increased fibrinolysis are the levels of D-dimers. Our study confirmed the notable increase in their levels during pregnancy. None of the investigated women had the level of D-dimers over the referent range during the first trimester. We detected that in $50 \%$ of subjects in the study group, the levels of D-dimers were within the referent range during the second trimester. The significant increase continued during the third trimester and achieved $95 \%$. Only $5 \%$ of our study group had 
this followed marker within normal range in the third trimester. The connection between hypercoagulable state due to increased D-dimers and the risk of thromboembolism is unknown. In fact, many authors found the increase in D-dimers to be a very sensitive marker of fibrinolytic activity following the formation of fibrin. Some authors suggest that the primary site of increased fibrinolytic activity may be the placental uterine interface, while the elevated levels are due to localized increase in fibrin formation $(14,15$, 16). Variations in protein $C$ activity and levels of antithrombin III were not significant. Clark reported that the activity of protein $\mathrm{C}$ seemed to be unaffected by pregnancy. Even if the protein $\mathrm{C}$ activity was diminishing during pregnancy, the values were within the normal non-pregnant range (17). Antithrombin III is the key inhibitor of blood coagulation. The consumption and lower levels of antithrombin III during pregnancy could have been caused by increased clotting-enzymes production. In their studies, Clark and Bremme found a modest decrease in antithrombin III levels at term $(12,17)$. Asakura found a slight decrease in antithrombin III during the third trimester and this decrease was associated with fibrinous layers found in placenta (18). Our study failed to detect a significant decrease in antithrombin III. The activity of protein $\mathrm{S}$ was significantly lower in the second trimester and was followed by a slight increase during the third trimester while almost reaching the first trimester levels. The coagulation changes are most significant in the third semester of pregnancy and are associated with hormonal changes, especially with the increasing levels of estrogen throughout pregnancy (19).

There is evidence showing that the women with factor $\mathrm{V}$ Leiden mutation might be at an increased risk of some severe obstetric complications. Maternal thrombophilia together with natural hemostatic changes during pregnancy shift the gentle balance towards thrombotic changes in placenta resulting in inadequate fetomaternal circulation and lead to decreased placental perfusion (20). Higher incidence of intrauterine growth restriction in our study group confirms the relationship between placental pathology and maternal thrombophilia. The etiology of intrauterine growth restriction is multifactorial, and thrombophilia might have an additional role. On the other hand several studies found no significant difference in birth weight deviation between FVL carriers and controls, indicating no elevated risk for intrauterine growth restriction (21). In 1998, Triplett and Harris identified a correlation between recurrent miscarriages and antiphospholipid antibodies syndrome in 1989, and in 1996, Sanson suggested that there is a connection between fetal loss and protein $\mathrm{C}$, protein $\mathrm{S}$ and antithrombin III deficiency $(22,23)$. Since then, numbers of studies have occurred trying to find the association between thrombophilia and abortion/stillbirth. Fetal chromosome defects have been suggested to be the most common reason for sporadic miscarriage, accounting for $50 \%$ of all miscarriages. The maternal factors seem to be more and more important in pregnancy outcome (24). It is now widely accepted that the recurrent miscarriage has a multifactorial etiology including prothrombotic state, structural uterine abnormalities, endocrinological defects, infections, immune dysfunctions or endometrial responsiveness. The meta-analysis including 31 studies regarding thrombophilic disorders and fetal loss showed that the factor V Leiden mutation was associated with early and late recurrent fetal losses and late non-recurrent fetal loss (25). Preeclampsia affects $5 \%$ of singleton pregnancies. Its etiology is still unclear. Preeclampsia is significantly associated with factor V Leiden mutation. There is also an increased tendency towards maternal complications such as disseminated intravascular coagulation (DIC) and acute renal failure. In our study, the prevalence of preeclampsia in FVL carriers was not higher than that in controls, and was in accordance with the five largest case-control studies and three prospective studies (26-33). Current evidence shows that there are similar vasculopathic changes in preeclampsia, IUGR, fetal loss and placental abruption. The etiology of placental abruption is multifactorial and thrombophilia might play its role. In our small study, only one placental abruption occurred. In the two of three largest retrospective studies and two prospective studies, they found no significant association between factor V Leiden mutation and placental abruption. Some authors suggest that the factor V Leiden carriers have less blood during delivery compared with non-carriers. Our study confirmed this suggestion (26, 33-36).

\section{Conclusion}

We confirmed that the hemostatic changes in pregnancy are significant and essential, and have the potential to cause adverse pregnancy outcome. The hypercoagulable state during pregnancy is considered to be physiological. Although most of the clotting factors rise during pregnancy, especially in the third trimester, there is no evidence of fibrinolytic overactivation. In our study, the carriership of factor V Leiden mutation did not affect the incidence of preeclampsia. Adverse pregnancy outcomes such as placental abruption were rare. Eclampsia, intrauterine fetal death and venous thromboembolism did not occur. Thromboprophylaxis may have influenced the prevalence of venous thromboembolism. The incidence of blood loss exceeding $1000 \mathrm{ml}$ during delivery was significantly higher in the control group. In patients with negative personal or family histories for thromboembolism the heterozygous factor V Leiden mutation is associated with a lower risk of thromboembolism in pregnancy and therefore neither the screening of all pregnant women nor the treatment of the low-risk carriers is recommended. The strict collaboration between obstetricians and hematologists in cases with thrombophilic etiology is the main key for successful pregnancy.

\section{References}

1. Nijkeuter M, Ginsberg JS, Huisman MV. Diagnosis of deep vein thrombosis and pulmonary embolism in pregnancy: a systematic review. J Thromb Haemost 2006; 4: 496-500.

2. Bourjeily G, Paidas M, Khalil H, Rosene-Montella K, Rodger M. Pulmonary embolism during pregnancy. Lancet 2010; 375 (9713): 500-512.

3. Martinelli I, Bucciarelli P, Mannucci PM. Thrombotic risk factors: Basic pathophysiology. Crit Care Med 2010; 38 (2): 3-9.

4. Bates SM, Greer IA, Pabinger I, Sofaer S, Hirsh J. Venous Thromboembolism, Thrombophilia, Antithrombotic Therapy, and Pregnancy American College of Chest Physicians Evidence-Based Clinical Practice Guidelines (8th Edition). Chest 2008; 133: 844-886. 
$140-144$

5. Trigg DE et al. Hormonal Influences on Hemostasis in Women. Semin Thromb Hemost 2011; 37 (1): 77-86.

6. Sibai B, Dekker G, Kupferminc M. Pre-eclampsia. Lancet 2005; 365 : 785-799.

7. Tikkanen M, Nuutila M, Hiilesmaa V, Paavonen J, Ylikorkala O. Clinical presentation and risk factors of placental abruption. Acta Obstet Gynecol Scand 2006; 85(6): 700-705.

8. Cox P, Marton T. Pathological assessment of intrauterine growth restriction. Best Pract Res Clin Obstet Gynaecol 2009; 23 (6): 751-764.

9. Burton GJ, Watson AL, Hempstock J, Skepper JN, Jauniaux E. Uterine glands provide histiotrophic nutrition for the human fetus during the first trimester of pregnancy. J Clin Endocrinol Metab 2002; 87: 2954-2959.

10. Burrows RF. Platelet disorders in pregnancy. Curr Opin Obstet Gynecol 2001; 13 (2): 115-119.

11. Sainio S, Kekomäki R, Riikonen S, Teramo K. Maternal thrombocytopenia at term - a population-based study. Acta Obstet Gynecol Scand 2000; 79: 744-749.

12. Bremme K. Haemostatic changes in pregnancy. Best Pract Res Clin Haematol 2003; 16 (2): 153-168.

13. O'Riordan MN, Higgins JR. Haemostasis in normal and abnormal pregnancy. Best Pract Res Clin Obstet Gynaecol 2003; 17: 385-396.

14. Dempfle CE. What role does the measurement of fibrinogen and its derivatives have in the diagnosis of disseminated intravascular coagulation? Blood Rev 2002; 16 (1): S23-S28.

15. Dempfle CE, Wurst M, Smolinski M, Lorenz S, Olenik D et al. Use of soluble fibrin antigen instead of D-dimer as fibrin-related marker may enhance the prognostic power of the ISTH overt DIC score. Thromb Haemost 2004; 91: 815-818.

16. Dempfle CE. Validation, calibration and specificity of quantitative Ddimer assay. Sem Vasc Med 2005; 5: 315-320.

17. Clark P, Brennand J, Conkie JA, McCall F, Greer IA, Walker ID. Activated protein $\mathrm{C}$ sensitivity. Protein $\mathrm{C}$, protein $\mathrm{S}$ and coagulation in normal pregnancy. Thromb Haemost 1998; 79: 1166-1170.

18. Asakura H, Obshita T, Suzukis S, Araki T. Correlation between grade III placenta and plasma antithrombin III activity in full term pregnancy. Gynecol Obstet Invest 2001; 52: 47-50.

19. Kher A, Bauersachs R, Nielsen JD. The management of thrombosis in pregnancy: Role of low-molecular-weight heparin. Thromb Haemost 2007; 97 (4): 505-513.

20. Redman CWG, Sargent IL. Preeclampsia, the Placenta and the Maternal Systematic Inflammatory Response - A Review. Placenta 2003; 24 (Suppl A): S21-S27.

21. Many A, Schreiber L, Rosner S, Lessing JB, Eldor A, Kupferminc MJ. Pathologic features of the placenta in women with severe pregnancy complications and thrombophilia. Obstet Gynecol 2001; 98: 1041-1044.

22. Triplet DA, Harris EN. Antiphospholipid antibodies and reproduction. Am J Reprod Immunol 1989; 21: 123-131.
23. Sanson BJ, Friedrich PW, Simioni P. The risk of abortion and stillbirth in antithrombin-, protein C-, and protein S-deficient women. Thromb Haemost 1996; 75: 387-388.

24. Sullivan EA, Silver RM, LaCoursiere DY, Porter TF, Branch DW. Recurrent fetal aneuploidy and recurrent miscarriage. Obstet Gynecol 2004; 104: 784-788.

25. Ray E, Kahn SR, David M, Shrier I. Thrombophilic disorders and fetal loss: a meta-analysis. Lancet 2003; 361: 901-908.

26. Dizon-Townson D, Miller C, Sibai B, Spong CY, Thom E, Wendel JrG. The relationship of the factor V Leiden mutation and pregnancy outcomes for mother and fetus. Obstet Gynecol 2005; 106: 517-524.

27. Clark P, Walker ID, Govan L, Wu O, Greer IA. The GOAL study: a prospective examination of the impact of factor V Leiden and $\mathrm{ABO}(\mathrm{H})$ blood groups on hemorrhagic an thrombotic pregnancy outcomes. $\mathrm{Br} \mathrm{J}$ Haematol 2007; 140: 236-240.

28. The GOPEC Consortium. Disentangling fetal and maternal susceptibility for pre-eclampsia: A British multicenter candidate--gene study. Am J Hum Genet 2005; 77: 127-131.

29. De Groot CJM, Bloemenkamp KWM, Duvenkot EJ, Helmerhorst FM, Bertina RM, Vander Meer F et al. Preeclampsia and genetic risk factors for thrombosis: A case--control study. Am J Obstet Gynecol 1999; 181: 975-980.

30. Kim YJ, Williamson RA, Murray JC, Andrews J, Pietscher JJ et al. Genetic susceptibility to preeclampsia: Roles of cytosine-to-thymine substitution at nucleotide 677 of the gene for methylenetetrahydrofolate reductase, 68-base pair insertion at nucleotide 844 of the gene for cystathionine $\beta$-synthase, and factor V Leiden mutation. Am J Obstet Gynecol 2001; 184: 1211-1217.

31. Mello G, Parretti E, Marozio L, Pizzi C, Lojacono A, Frusca T et al. Thrombophilia is significantly associated with severe preeclampsia. Hypertension 2005; 46: 1270-1274.

32. O'Shaughnessy KM, Fu B, Ferraro F, Lewis I, Downing S, Morris NH. Factor V Leiden and thermolabile methylenetetrahydrofolate reductase gene variants in an East Anglian preeclampsia cohort. Hypertension 1999; 33: 1338-1341.

33. Lindquist P, Dahlbäck B, Maršál K. Thrombotic risk during pregnancy: A population study. Obstet Gynecol 1999; 94: 595-599.

34. Jääskeläinen E, Tiovonen S, Romppanen EL, Helisalmi S, KeskiNisula L, Punnonen K, Heinonen S. M385T polymorphism in the factor $\mathrm{V}$ gene, but not Leiden mutation, is associated with placental abruption in Finnish women. Placenta 2004; 25: 730-734.

35. Procházka M, Happach C, Maršál K, Dahlbäck B, Lindquist PG. Factor V Leiden in pregnancies complicated by placental abruption. BJOG 2003; 110: 462-466.

36. Procházka M, Lubušký M, Slavík L, Hrachovec P, Zielina P, Kudela M, Lindquist PG. Frequency of selected thrombophilias in women with placental abruption. Aust N Z J Obstet Gynaecol 2007; 47 (4): 297-301.

Received March 31, 2012. Accepted October 27, 2013. 